\title{
ARTICLE \\ Sphingomyelin synthase 2 facilitates M2-like macrophage polarization and tumor progression in a mouse model of triple-negative breast cancer
}

\author{
Yan Deng ${ }^{1}$, Jia-chun $\mathrm{Hu}^{1}$, Shu-hua He${ }^{1}$, Bin Lou ${ }^{1}$, Ting-bo Ding ${ }^{2}$, Jin-tong Yang ${ }^{3}$, Ming-guang $\mathrm{Mo}^{3}$, De-yong Ye ${ }^{3}$, Lu Zhou ${ }^{3}$,
} Xian-cheng Jiang ${ }^{4}$, Ker $\mathrm{Yu}^{1}$ and Ji-bin Dong ${ }^{1,5}$

High infiltration of M2-polarized macrophages in the primary tumor indicates unfavorable prognosis and poor overall survival in the patients with triple-negative breast cancer (TNBC). Thus, reversing M2-polarized tumor-associated macrophages in the tumors has been considered as a potential therapeutic strategy for TNBC. Sphingomyelin synthase 2 (SMS2) is the key enzyme for sphingomyelin production, which plays an important role in plasma membrane integrity and function. In this study we investigated whether SMS2 inhibitor or SMS2 gene knockout could reduce macrophages M2 polarization and tumor progression in a mouse model of TNBC. We showed that SMS2 mRNA expression was linked to immunosuppressive tumor microenvironment and poor prognosis in TNBC patients. The knockout of SMS2 or application of 15w (a specific SMS2 inhibitor) markedly decreased the generation of M2-type macrophages in vitro, and reduced the tumor weight and lung metastatic niche formation in a 4T1-TNBC mouse model. We further demonstrated that the in vivo antitumor efficacy of $15 \mathrm{w}$ was accompanied by a multifaceted remodeling of tumor immune environment reflecting not only the suppression of M2-type macrophages but also diminished levels of regulatory $\mathrm{T}$ cells and myeloid-derived suppressor cells leading to a dramatically improved infiltration of antitumor CD8 ${ }^{+} \mathrm{T}$ lymphocytes. Collectively, our results reveal a novel and important role of SMS2 in the protumorigenic function and may offer a new strategy for macrophage-targeted anticancer therapy.

Keywords: triple-negative breast cancer; macrophages; M2 polarization; sphingomyelin synthase 2; 15w; immunosuppressive cells; tumor microenvironment

Acta Pharmacologica Sinica (2021) 42:149-159; https://doi.org/10.1038/s41401-020-0419-1

\section{INTRODUCTION}

Triple-negative breast cancer (TNBC) is an aggressive subtype of breast cancer that accounts for $15 \%$ of all breast carcinomas [1, 2]. TNBC is defined by the absence of estrogen, progesterone, and HER2 receptors. Therefore, patients with TNBC are not eligible for hormone or anti-HER2 therapy [3]. The standard care for TNBC patients is neoadjuvant chemotherapy (NAC) [4]. While NAC is effective in some TNBC patients, $\sim 30 \%-50 \%$ of patients eventually develop tolerance, leading to poor overall survival [5-7]. The response of neoplastic cells to chemotherapy is not solely dependent on the genomic aberrations they harbor but is also regulated by the tumor microenvironment (TME) [8]. Identification of therapeutic targets in the TME to initiate antitumor immune responses could be a novel and effective strategy for improving long-term patient survival $[9,10]$.

Macrophages are one of the dominant components in the breast cancer microenvironment and are considered regulators in multiple breast cancer processes based on their various phenotypes [11-13]. Fully polarized macrophages are called M1 (classically activated) or M2 (alternatively activated) macrophages, which are the extremes of a continuum of functional states [14]. Tumor-associated macrophages (TAMs) that are highly enriched in the TME are driven by tumors and stromal cell-derived cytokines to acquire an M2 phenotype [14-16]. These M2 macrophages promote tumor development through multiple pathways [17]. Various preclinical studies have demonstrated that inhibiting the recruitment of macrophages or reprogramming their phenotype to limit M2 polarization can improve treatment response in mouse models [18-20]. High infiltration of M2-polarized macrophages in the primary tumor indicates unfavorable prognosis [21, 22] and poor overall survival in TNBC patients [23-25]. Therefore, reversing the M2 polarization of TAMs in tumors could be considered a potential therapeutic strategy in TNBC [26].

Sphingomyelin (SM) is an important component in plasma membrane lipid rafts, where many receptors and transporters are located [27-29]. Plasma membrane-bound sphingomyelin synthase 2 (SMS2) is involved in maintaining the SM level on the membrane [30, 31]. Studies have indicated that peritoneal macrophages from SMS2 knockout (SMS2-KO) mice exhibit increased cholesterol efflux and decreased inflammatory

\footnotetext{
${ }^{1}$ Department of Pharmacology and Biochemistry, School of Pharmacy, Fudan University, Shanghai 201203, China; ${ }^{2}$ Experiment \& Teaching Center, School of Pharmacy, Fudan

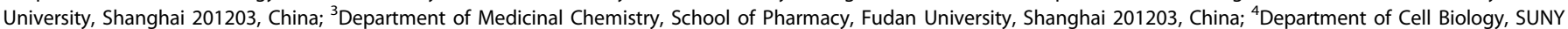
Downstate Medical Center, Brooklyn, NY 11203, USA and ${ }^{5}$ Shanghai Engineering Research Center of Immunotherapeutics, Fudan University, Shanghai 201203, China Correspondence: Ker Yu (keryu@fudan.edu.cn) or Ji-bin Dong (jbdong@shmu.edu.cn)
}

Received: 21 January 2020 Accepted: 14 April 2020

Published online: 25 May 2020 
responses [32]. SMS2 deficiency attenuated NF-KB activation under the stimulation of endotoxin and palmitate, suggesting a proinflammatory role of SMS2 in macrophages [33]. We consider the possibility that SMS2 may also play a role in the phenotype and function of TAMs in the TME.

In the present study, we systematically examined the effect of SMS2 inhibition or SMS2 gene knockout on macrophage M2 polarization. We also investigated the antitumor efficacy of an SMS2-selective inhibitor on orthotopic tumor growth and lung metastatic niche formation in a mouse model of 4T1 breast cancer.

\section{MATERIALS AND METHODS}

Chemicals and inhibitors

A selective SMS2 inhibitor designated $15 \mathrm{w}$ was synthesized as described [34]. All other chemicals used for general buffers and assays were obtained from Sinopharm Chemical Reagent (Shanghai, China) unless specified.

\section{Bioinformatics analysis}

The expression of SMS2 in normal and cancerous breast tissues was analyzed in the Finak Breast Statistics dataset in Oncomine (https://www.oncomine.org). Affymetrix array data for a breast cancer cohort of 522 patients were acquired from TCGA within the Genomic Data Common data portal [35] to examine the association between SMS2 expression and biomarker expression of M2 macrophages. Survival curves were analyzed by using the online tool Kaplan-Meier plotter (https://kmplot.com).

Cell lines and cell culture

4T1 and MDA-MB-231 cells were obtained from American Type Culture Collection. Luciferase-labeled 4T1 cells (4T1-luc) were kindly provided by Prof. Qi-zhi Zhang of Fudan University School of Pharmacy [36]. Cells were cultured in RPMI-1640 containing $10 \%$ fetal bovine serum (FBS) (Gibco).

\section{SMS2 knockout mice and mouse models of breast cancer} All animal studies were performed under protocols approved by IACUC of Fudan University School of Pharmacy. SMS2-KO C57BL/6 mice were kindly donated by Prof. Xian-cheng Jiang [32]. SMS2WT/KO mice were genotyped using PCR with specific primers: for WT: forward, 5'-GTGGCGGACAATGGATATCATAGAGACAGC-3' and reverse, 5'-GATAAGGTCTTGGGTITGCCCTTGCC-3'; for KO: forward, 5'-GCCAGAGGCCACTTGTGTAGC-3' and reverse, 5'-GATAAGGTCT TGGGTTTGCCCTTGC-3'.

Six-week-old female BALB/c mice were purchased from Charles River Laboratory (Beijing, China) and housed in an approved animal care facility at Fudan University. The mice were randomly assigned to two groups (eight mice per group) and orally administered $15 \mathrm{w}(20 \mathrm{mg} / \mathrm{kg})$ or vehicle $(0.5 \%$ sodium carboxymethylcellulose). The plasma SM level was detected by a mouse SM ELISA kit (mlbio; SU-B20566). Two weeks after obvious reduction of SM level detected in $15 \mathrm{w}$-treated mice, the mice were implanted with $4 \mathrm{~T} 1$ cells for breast cancer modeling.

For the orthotopic tumor model, $2 \times 10^{5} 4 \mathrm{~T} 1$ cells suspended in $100 \mu \mathrm{L}$ PBS were inoculated in the fourth mammary fat pads (MFPs) of the BALB/C mice. Tumor size was measured with a caliper, and tumor volumes were calculated by using the equation: tumor volume $=$ length $\times$ width $^{2} \times 0.5$. All mice were sacrificed, and tumors were harvested on day 14 before distant metastasis.

For the experimental metastasis model, 4T1-luc cells were intravenously injected into the tail vein. The fluorescence intensity of 4T1-luc cells was detected by bioluminescence imaging (BioRad) on day 14. One week later, the mice were dissected, and lung tissues were subjected to histological analysis.
Monocyte isolation and bone marrow-derived macrophage differentiation

Six-week-old C57BL/6 mice were sacrificed using cervical dislocation after euthanasia with urethane. Then, femurs and tibias were removed aseptically. The bone cavities were flushed using a 25G needle filled with RPMI-1640. Bone marrow cells were collected and cultured in RPMI-1640 supplemented with 10\% heatinactivated FBS (Gibco) and $20 \mathrm{ng} / \mathrm{mL}$ M-CSF (PeproTech; 31502) for 7 days, and the medium was replaced every 3 days. The phenotypes of mature macrophages were analyzed by flow cytometry.

SMS activity measurement

Bone marrow-derived macrophages (BMDMs) at $5 \times 10^{5}$ cells $/ \mathrm{mL}$ were pretreated with various doses of $15 \mathrm{w}$ for $2 \mathrm{~h}$. The cells were incubated with $0.1 \mathrm{mg} / \mathrm{mL}$ C6-NBD-ceramide (Sigma-Aldrich) for 2 $\mathrm{h}$ before the supernatants were extracted in chloroform:methanol solution (2:1 by volume). The cells were centrifuged at $3000 \times g$ for $10 \mathrm{~min}$, and the lower phase was isolated, dried under a $\mathrm{N}_{2}$ stream, and redissolved in $100 \mu \mathrm{L}$ methanol. NBD-SM was detected by using HPLC as reported by Huang et al. [37].

Preparation of conditioned medium

To obtain TNBC cell-conditioned medium (cell-CM), 4T1 or MDA-MB231 cells were cultured to reach $90 \%$ confluence, and then replaced with serum-free fresh medium for $24 \mathrm{~h}$ of incubation. The supernatants were collected and centrifuged at $5000 \mathrm{r} / \mathrm{min}$ for $10 \mathrm{~min}$, and then filtered through $0.22 \mu \mathrm{m}$ nylon syringe filters. The medium was stored at $-80^{\circ} \mathrm{C}$ for the following assays. For macrophage-CM, mature macrophages were cultured and stimulated with $20 \mathrm{ng} / \mathrm{mL}$ IL-4 with or without $15 \mathrm{w}$ for $48 \mathrm{~h}$. Subsequently, the supernatant was replaced with serum-free medium for $24 \mathrm{~h}$. Then, the medium was collected and stored at $-80^{\circ} \mathrm{C}$.

\section{RNA isolation and RT-qPCR}

Total RNA was isolated and purified by TRIzol reagent (Invitrogen; 15596018) according to the standard protocol. Reverse transcription was performed using PrimeScript ${ }^{T M}$ RT Master Mix (Takara Bio; RR036B) for $500 \mathrm{ng}$ of total RNA. Then, quantitative real-time PCR was performed using TB Green Premix Ex Taq $^{\text {TM }}$ II (Takara Bio; RR820L). The relative mRNA levels of specific genes were normalized to the $\beta$-actin level. The following primers were used: mouse CD206: forward, 5'-CTCTGTTCAGCTATTGGACGC-3' and reverse, 5'-CGGAATTTCTGGGATTCAGCTTC-3'; Arg1: forward, 5'-TC CCGCTCTAAGCACACCA-3' and reverse, 5'-CCGGTTCCGTTTGCTCT CC-3'; TGF- $\beta$ : forward, 5'-AGACCACATCAGCATTGAGTG-3' and reverse, 5'-GGTGGCAACGAATGTAGCTGT-3'; and $\beta$-actin: forward, 5'-ATGGTTGACGATGAGCTGACT-3' and reverse, 5'-GACTCCGTGTT CAATGGGATAC-3'.

\section{Western blotting}

BMDMs were stimulated with $20 \mathrm{ng} / \mathrm{mL} \mathrm{IL-4}$ (PeproTech; 214-14) or TNBC cell-CM supplemented with $10 \%$ FBS for $48 \mathrm{~h}$, and then lysed on ice by directly adding NuPAGE ${ }^{\mathrm{TM}}$ LDS Sample Buffer (Invitrogen, NP0007) to cell culture plates. Protein samples were heated at $70{ }^{\circ} \mathrm{C}$ for $10 \mathrm{~min}$, separated by SDS-PAGE, and electrotransferred to a PVDF membrane (Merck Millipore). The primary antibodies used in the study were Arg1 (93668, CST), P-Akt (4060S, CST), Akt (4691S, CST), P-S6 (2268-1, EPITOMICS), and S6 (2217, CST). Protein bands were visualized using an ECL detection kit (Merck Millipore), and images were obtained using a chemiluminescence detection system (Bio-Rad).

Flow cytometry analysis

Macrophages were incubated with anti-mouse F4/80 and antimouse CD11b antibodies. Subsequently, the cells were fixed in fixation buffer (BioLegend) and washed with IC permeation buffer (BioLegend). Cells were incubated with anti-mouse CD206 to 
detect M2-polarized macrophages. All antibodies were incubated with cells at $4{ }^{\circ} \mathrm{C}$ for $30 \mathrm{~min}$ in the dark. Antibodies for cell staining were PE-Cyanine7 anti-mouse F4/80 (eBioscience; 25-4801-82), PE anti-mouse CD86 (BioLegend; 105015), and APC anti-mouse CD206 (eBioscience; 17-2069-42).

Cytokine analysis

BMDMs were stimulated with CM collected from 4T1 and MDAMB-231 cells in the presence or absence of $15 \mathrm{w}$ for $48 \mathrm{~h}$, and then the media were exchanged with serum-free media after culturing cells for $24 \mathrm{~h}$. The cell culture supernatant was harvested, and the concentrations of TGF- $\beta$ and IL-10 were measured by using an ELISA kit (ExCell Bio).

Cell migration assay

In six-well plates, $2 \times 10^{5} 4 \mathrm{~T} 1$ cells per well were plated and cultured with CM from macrophages for $48 \mathrm{~h}$. After that, a migration assay was performed in a 24-well plate using $8-\mu \mathrm{m}$ pore size Transwell inserts (Corning, 3422). The 4T1 cells were seeded in the upper chamber, while 1640 medium containing $10 \%$ FBS was added to the well of the plate. After incubation for $12 \mathrm{~h}$, cells that migrated to the reverse face of the membrane were fixed and stained with $0.2 \%$ crystal violet. Observation fields were randomly selected and captured using a Nikon inverted microscope.

Immunohistochemistry and immunofluorescence

Tumor tissues and lung tissues were dissected for paraffin embedding and sectioning (Biossci Biotechnology, China). Terminal deoxynucleotidyl transferase dUTP nick-end labeling (TUNEL) staining and hematoxylin and eosin (H\&E) staining were performed following the manufacturer's protocol. The expression of some proteins was analyzed by immunohistochemistry (IHC) and immunofluorescence (IF) staining. Antibodies used for IHC and IF were F4/80 (Baiaosi bioscience; BA9048), CD206 (Abcam; ab64693), CD31 (Baiaosi bioscience; BA9001), CD31 (Abcam/ ab182981), alpha-SMA (Boster/BM0002), FOXP3 (CST/98377), CD4 (Abcam/AB183685), PE-CD11b (BioLegend; 101207), and FITC-Gr1 (Elabscience; E-AB-F1120UC).

Statistical analysis

Data are presented as the mean \pm standard deviation except for in vivo data, which are presented as the mean \pm standard error of the mean. Statistical analysis was performed using Prism 6 (GraphPad Software, San Diego, CA, USA) and analyzed by twotailed Student's $t$ tests to determine the significance of differences between two groups. $P$ values $<0.05$ were considered statistically significant; ${ }^{* *} P<0.01 ;{ }^{* *} P<0.001 ;{ }^{* * *} P<0.0001$.

\section{RESULTS}

Elevated SMS2 expression is associated with an enriched tumorassociated macrophage signature and a worse prognosis in basallike/TNBC patients

We compared SMS2 mRNA levels between invasive breast carcinoma tissues and adjacent normal tissues based on the Oncomine database to investigate the potential association of SMS2 expression with invasive breast cancer. We found that SMS2 is highly expressed in breast carcinoma patients (Fig. 1a). Next, we examined the association between SMS2 expression and biomarkers for M2 macrophages based on transcriptome analysis of 522 breast tumor samples from TCGA. The median value of SMS2 expression was used as a cut-off to categorize tumor samples into "SMS2-high" and "SMS2-low" groups. We found that SMS2 mRNA is positively correlated with the mRNA ratio of CD206 and CD68, indicating that the high expression of SMS2 is likely to predict high infiltration of M2 macrophages in breast cancer (Fig. 1b). We also analyzed signature genes for immunosuppressive cells, including those for regulatory
T cells (Tregs) and CD11b and CD33 for myeloid-derived suppressor cells (MDSCs). SMS2 expression was positively associated with high infiltration of Tregs and MDSCs (Fig. 1c). To identify SMS2 expression disparity in different molecular subtypes of breast cancer, we classified 522 breast tumors as "basal-like" or "luminal-like" based on the mRNA expression ratio (KRT5 + KRT14)/(KRT8 + KRT18) [38]. We found that SMS2 expression was much higher in patients with basal-like breast cancer, which represents the majority of TNBC patients and is associated with a worse prognosis than luminal-like breast cancer (Fig. 1d, e).

SMS2 gene knockout attenuates macrophage M2 polarization We next sought to explore the role of SMS2 in regulating macrophage polarization toward the M2 phenotype in the BMDM system. We prepared monocytes from the bone marrow of SMS2 wild-type (SMS2-WT) and SMS2-KO mice that were verified by genotyping (Fig. 2a). Fully differentiated macrophages were quantified by flow cytometry analysis of $\mathrm{F} 4 / 80$, a pan-macrophage marker. An F4/80 expression rate over $90 \%$ indicated successfully differentiated macrophages that were suitable for further experiments (Fig. 2b). We stimulated BMDMs with IL-4 $(20 \mathrm{ng} / \mathrm{mL})$ for $48 \mathrm{~h}$ to induce M2 macrophages. Compared with the BMDMs from SMS2-WT, the SMS2-KO BMDMs exhibited a significantly decreased overall percentage of $\mathrm{F} 4 / 80^{+} \mathrm{CD} 206^{+}$double-positive cells and mean fluorescence intensity (MFI) of CD206 (Fig. 2C). Similar analysis of $\mathrm{F} 4 / 80^{+} \mathrm{CD} 86^{+}$double-positive $\mathrm{M} 1$ macrophages did not reveal a significant difference between the two cell populations (Fig. S1). We further analyzed the mRNA expression profile of classical M2 markers by real-time PCR and found that the mRNA levels of Arg1, CD206, YM1, and TGF- $\beta$ were all significantly induced in response to IL-4-stimulated $\mathrm{M} 2$ polarization, while these effects were greatly reduced in the SMS2-KO BMDMs compared with controls (Fig. 2d). Consistent with the mRNA results, Arg1 protein expression in SMS2-KO BMDMs was also dramatically decreased compared with that in SMS2-WT BMDMs (Fig. 2e). These results support a role for SMS2 in M2 polarization and BMDM function.

Pharmacological inhibition of SMS2 decreases M2 polarization To further confirm the role of SMS2 in macrophage M2 polarization, we examined the effect of the SMS2-selective inhibitor 15w; its chemical structure is shown in Fig. 3a. As shown in Fig. 3b, 15w exerted dose-dependent inhibition of the SMS2 enzyme in BMDMs with an IC $\mathrm{C}_{50}$ value of $3.0 \mu \mathrm{M}$. We chose $10 \mu \mathrm{M}$ $15 \mathrm{w}$ for further experiments based on a threefold increase in the $\mathrm{IC}_{50}$ and the lack of general cytotoxicity at this dose. BMDMs were pretreated with $15 \mathrm{w}$ for $1 \mathrm{~h}$ and then $\mathrm{M} 2$ polarization was stimulated with IL-4. In line with previous results of SMS2-KO, 15w significantly reduced the percentage of $\mathrm{F} 4 / 80^{+} \mathrm{CD} 206^{+} \mathrm{BMDMs}$ and the MFI of CD206 (Fig. 3c). Similarly, 15w treatment also significantly suppressed the upregulation of $\mathrm{M} 2$ markers at the mRNA and protein levels in the process of M2 activation (Fig. 3d, e). Thus, targeting SMS2 either via gene depletion or enzyme inhibition consistently and efficiently reduces $\mathrm{M} 2$ polarization and signaling function in BMDMs.

SMS2 inhibitor reduces tumor cell-conditioned medium (cell-CM)induced M2 macrophage polarization

To mimic the effect of tumor cells on TAM polarization in vitro, we cultured BMDMs in CM collected from the cell culture of two representative TNBC cell lines, mouse $4 \mathrm{~T} 1$ and human MDA-MB231. The BMDMs cultured in CM from both cell types displayed an elongated and stretched morphology consistent with M2 macrophages. We then cultured BMDMs in the above-described CM without or with $15 \mathrm{w}$ for 2 days. As shown in Fig. 4a, b, while the CD206 MFI was dramatically increased in response to incubation with TNBC-CM from both cell types, $15 \mathrm{w}$ significantly attenuated the increase in CD206. Moreover, there was only a minor induction 
a

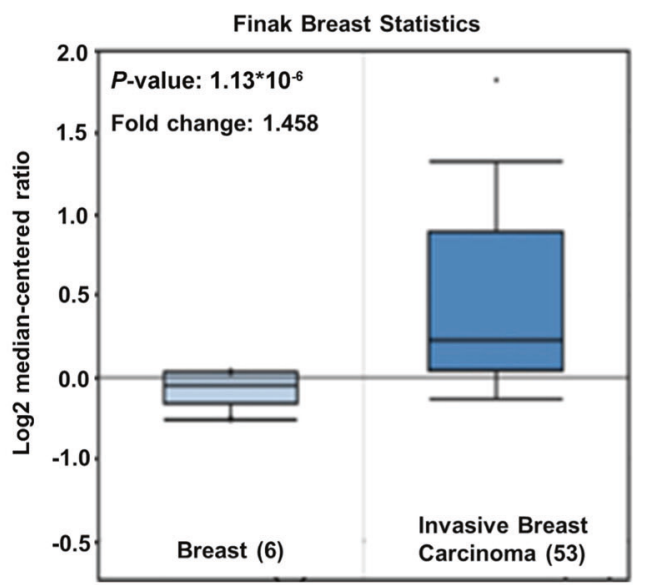

b

CD206/CD68

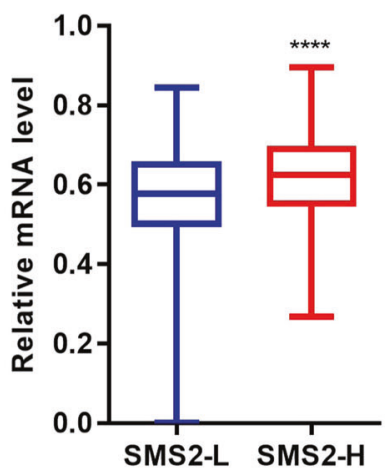

C

FOXP3

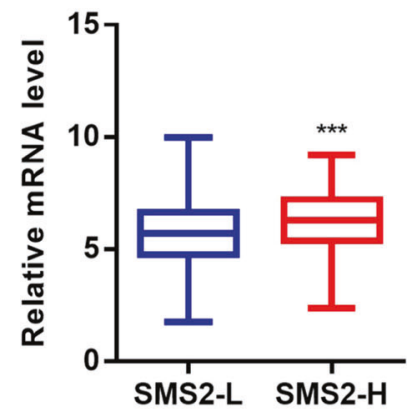

d

SMS2

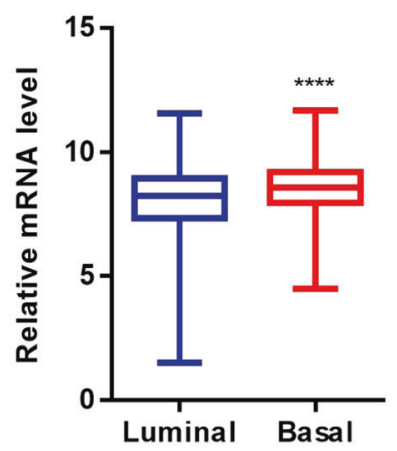

CD11b

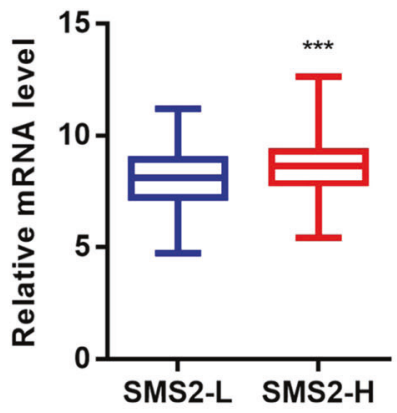

CD33

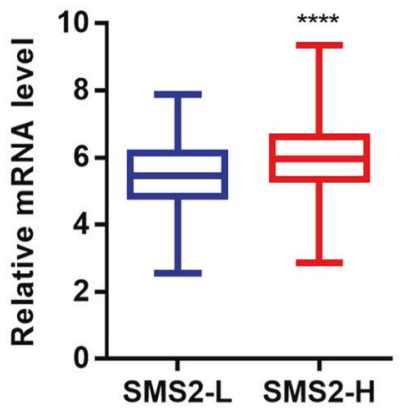

SMS2

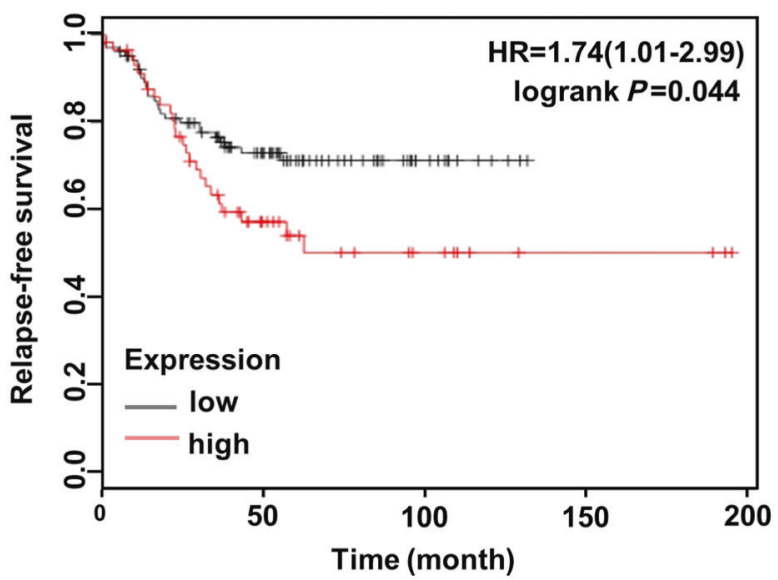

Fig. 1 High expression of SMS2 correlates with poor prognosis in patients with basal-like breast cancer. a SMS2 is highly expressed in breast cancer patients based on Oncomine data. b, c A total of 522 TCGA samples were defined as SMS2-high or SMS2-low according to the median value of SMS2 expression. A positive relationship between SMS2 and biomarkers of immunosuppressive cells was found in SMS2-high patients. Basal-like breast cancer patients showed a higher SMS2 level (d) and a worse prognosis (e) than luminal-like breast cancer patients. ${ }^{* * *} P<0.001 ; * * * * P<0.0001$.

of the CD86 MFI in the TNBC-CM-incubated BMDMs, indicating a limited increase in M1 polarization. Next, we analyzed TAM-related cytokines, such as IL-10 and TGF- $\beta$, to examine the functional participation of SMS2 in CM-induced M2 polarization. Treatment with 15 w significantly reduced the CM-stimulated secretion of IL10 and TGF- $\beta$ in BMDMs cultured in CM from 4T1 cells (Fig. 4c) or CM from MDA-MB-231 cells (Fig. 4d). Taken together, these results indicate that the SMS2 inhibitor can effectively reduce TNBC cell- induced tumor-associated M2 macrophage polarization but not M1 macrophage polarization.

Antimetastatic and immunomodulatory effects of the SMS2 inhibitor in a mouse breast cancer lung metastasis model To examine the effect of SMS2 on macrophage-mediated tumor cell migration, we polarized BMDMs under $\mathrm{M} 2$ conditions without or with $15 \mathrm{w}$ and collected conditioned media as shown in the 
a

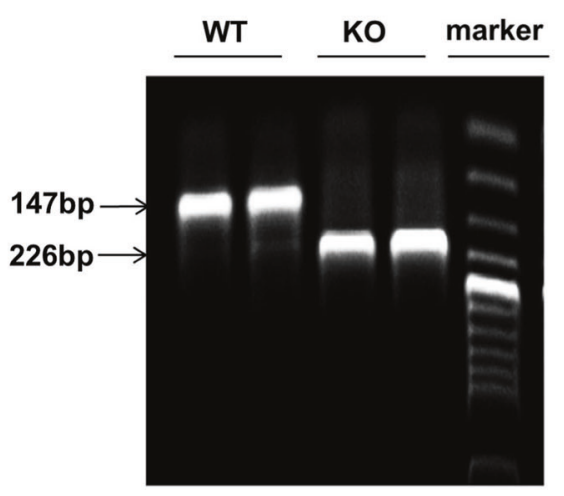

b

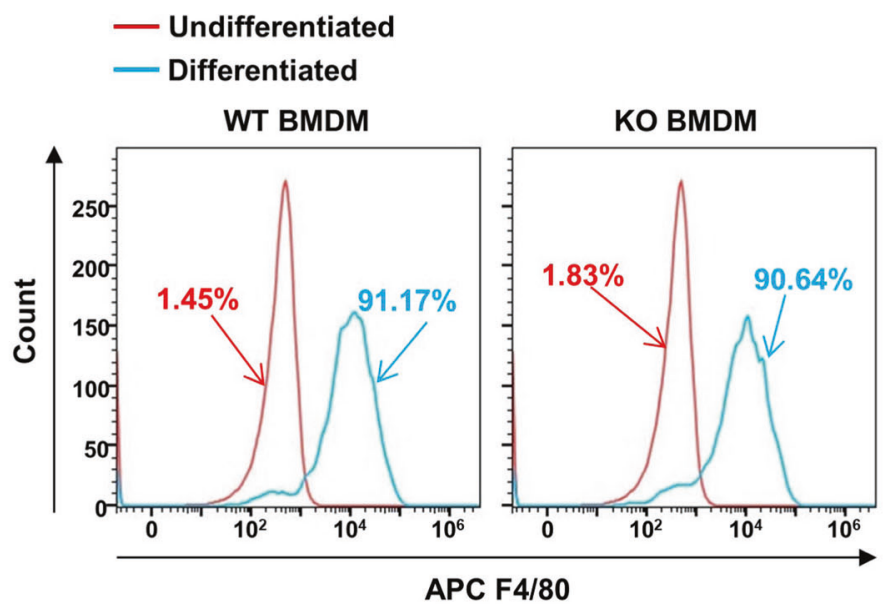

c

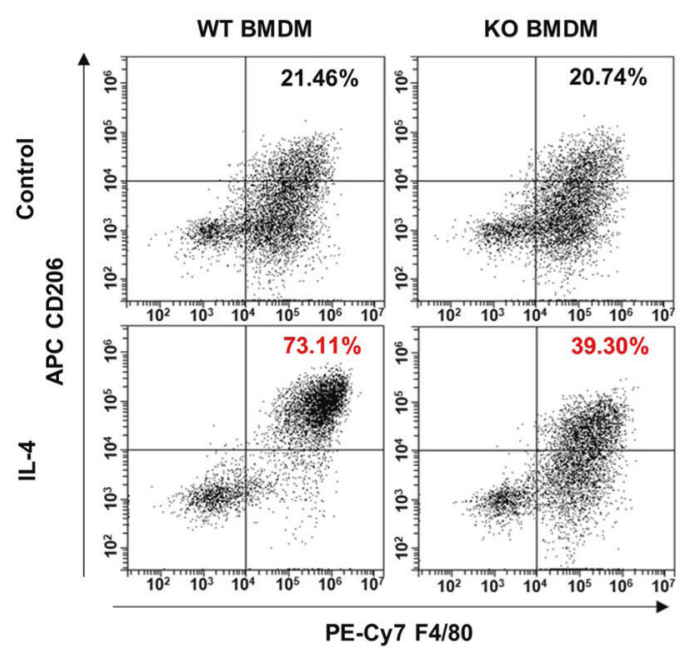

e

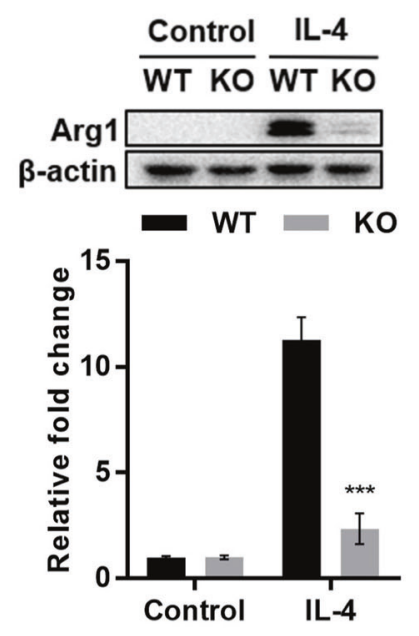

d

$$
\begin{array}{r}
\text { WT } \\
\text { KO }
\end{array}
$$
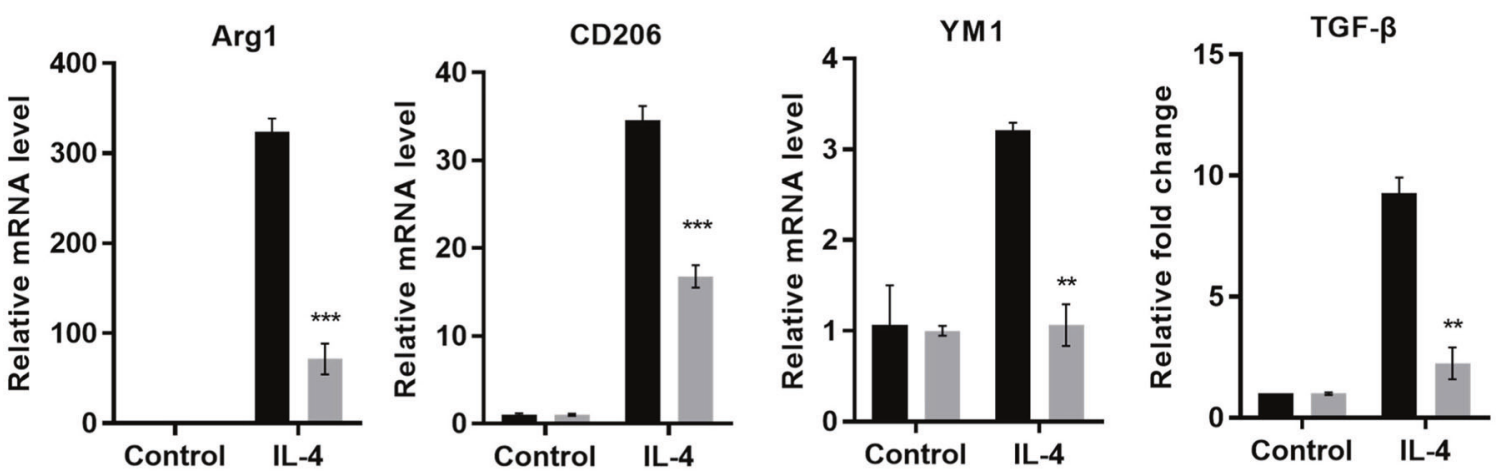

Fig. 2 SMS2-KO impairs the M2 polarization of bone marrow-derived macrophages. a Mouse genotyping was verified using PCR. Flow cytometry analysis of pan-macrophage marker F4/80 (b) or the M2 marker F4/80+ CD206+ (c), which showed no difference in overall differentiation but reduced M2 polarization in SMS2-KO BMDMs. d Real-time PCR analysis of the indicated gene in BMDMs under the indicated conditions. e Immunoblotting of SMS2-WT and SMS2-KO BMDMs after $48 \mathrm{~h}$ of M2 polarization with IL-4. Data are presented as the mean \pm SD of three representative experiments. ${ }^{* *} P<0.01 ;{ }^{* *} P<0.001$. 
a<smiles>Fc1ccc(Cl)c(COc2cccc3onc(Nc4cccnc4)c23)c1</smiles>

$15 w$ b

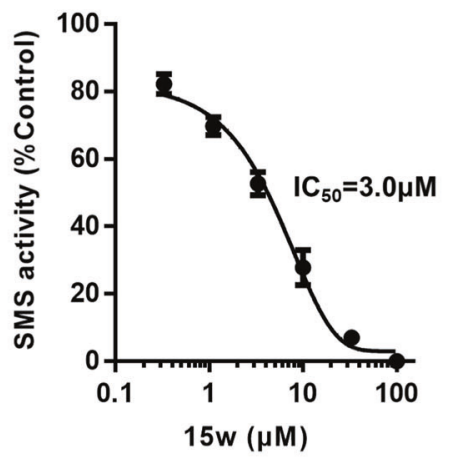

C
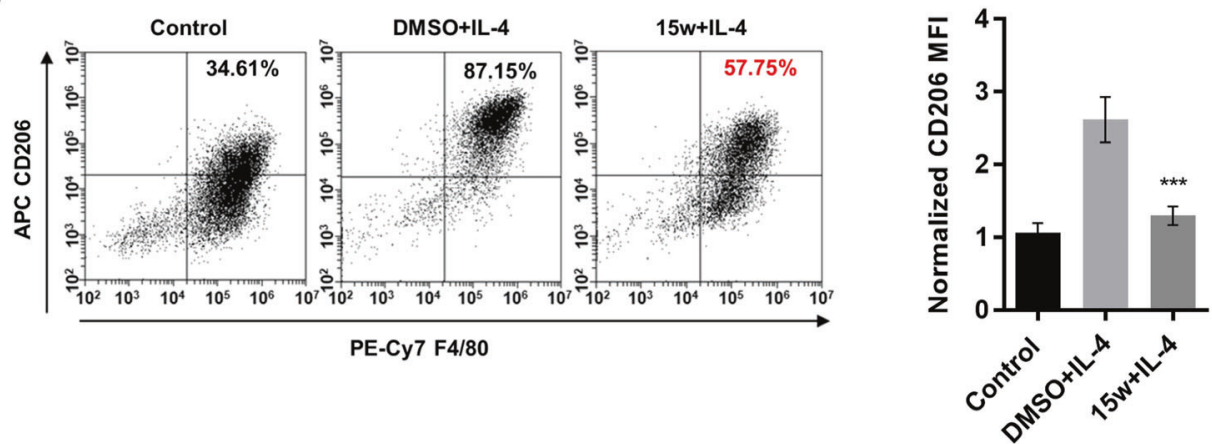

d
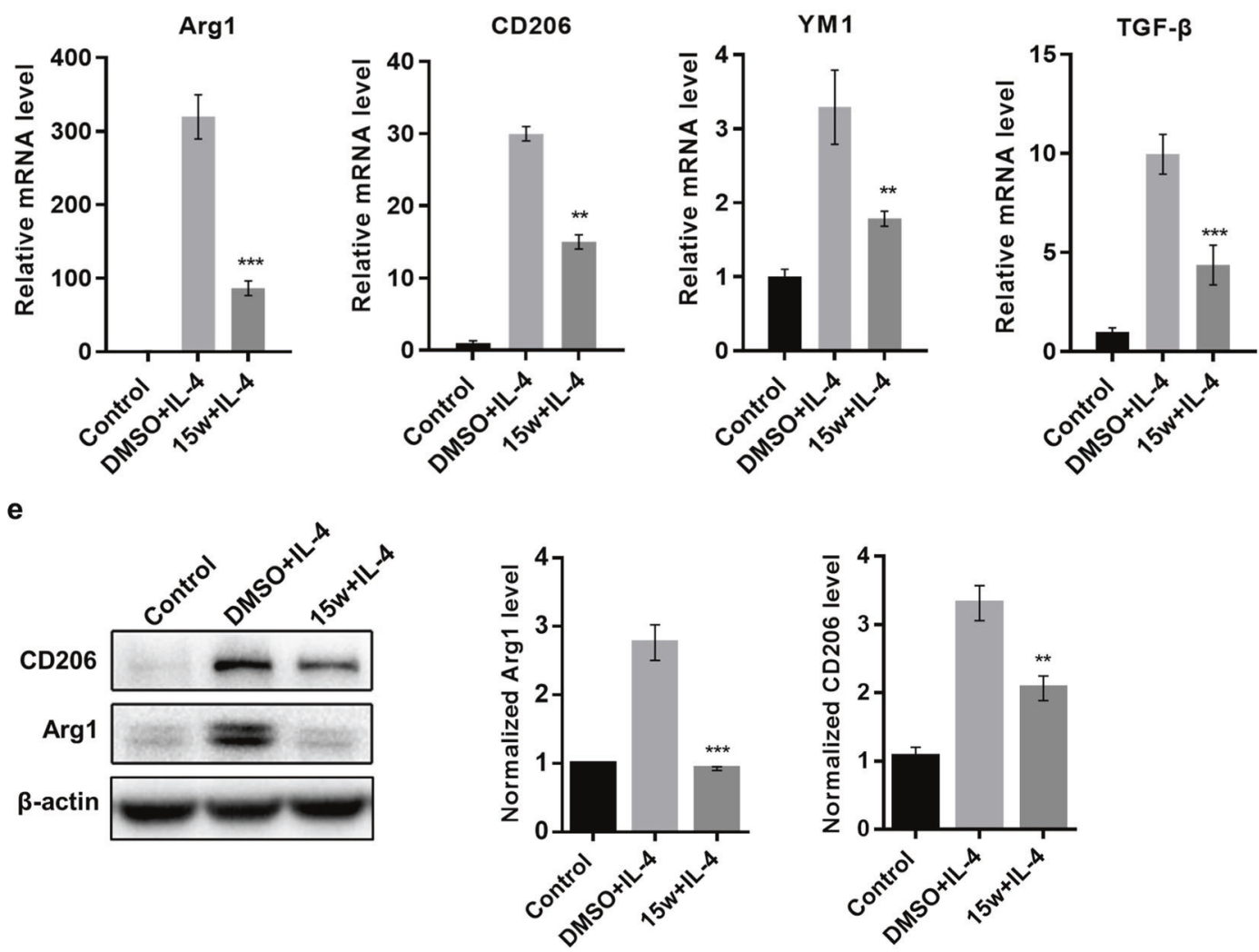

Fig. 3 SMS2-selective inhibitor $15 w$ attenuates M2 macrophage polarization. a The chemical structure of compound $15 \mathrm{w}$. Molecular weight: 369.78. Molecular formula: $\mathrm{C}_{19} \mathrm{H}_{13} \mathrm{CIFN}_{3} \mathrm{O}_{2}$. b HPLC measurements of SMS2 enzyme inhibition by $15 \mathrm{w}$ in BMDMs. c Flow cytometry quantification of M2 macrophage polarization without or with $15 \mathrm{w}$. d Real-time PCR was performed $24 \mathrm{~h}$ after IL-4-stimulated M2 polarization in BMDMs. e Immunoblotting of the indicated BMDMs $48 \mathrm{~h}$ after IL-4 stimulation. Data are presented as the mean \pm SD of three representative experiments. ${ }^{* *} P<0.01 ;{ }^{* * *} P<0.001$. 

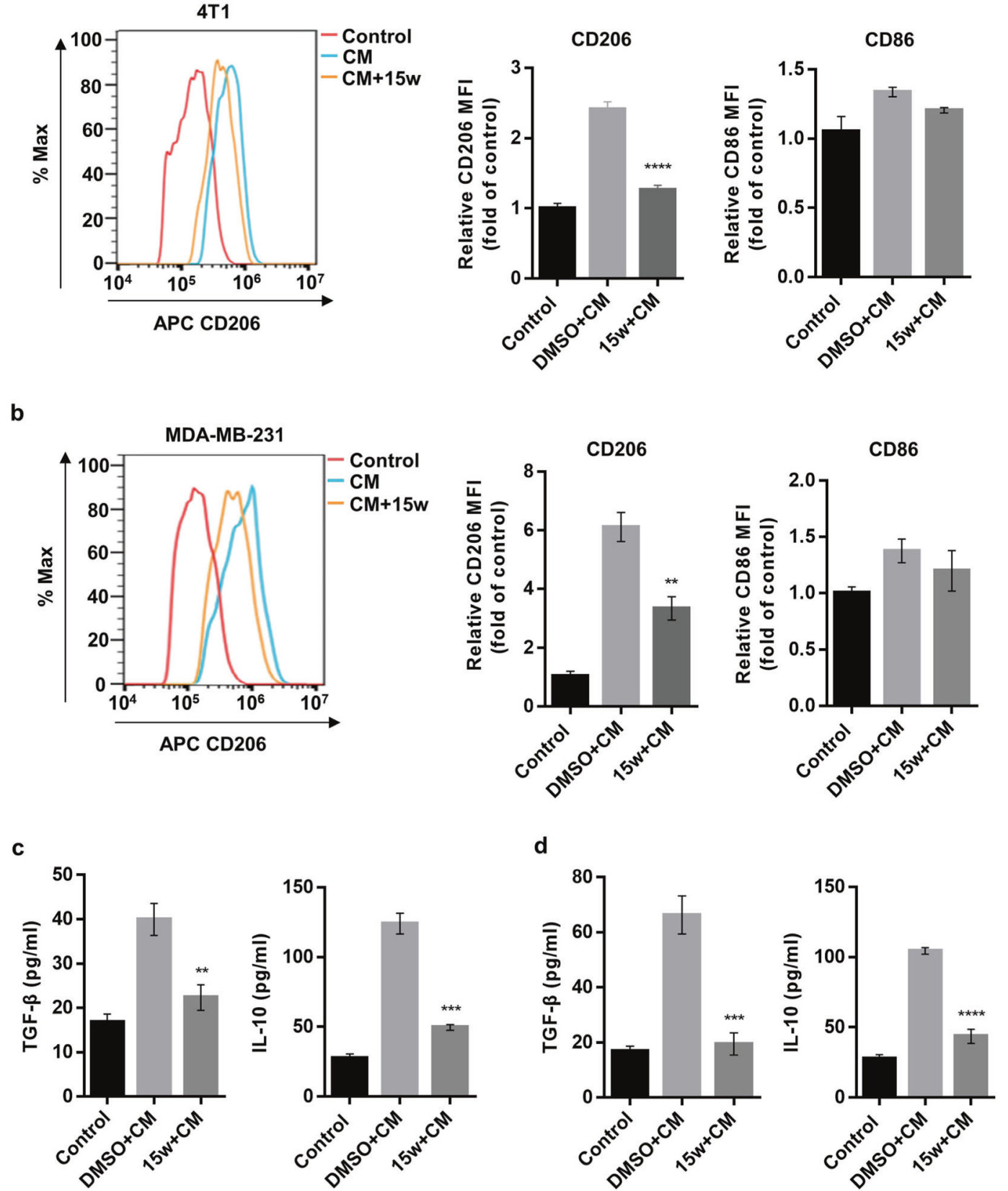

Fig. 4 SMS2 inhibitor partially prevents TNBC cell-induced macrophage M2 polarization. $\mathbf{a}$, b Conditioned medium (CM) from 4T1 or MDAMB-231 cells was used to culture BMDMs without or with $15 \mathrm{w}$ for $24 \mathrm{~h}$, and then the cells were analyzed by flow cytometry. c, $\mathbf{d}$ The levels of secreted cytokines were measured by ELISA $48 \mathrm{~h}$ later. Data are presented as the mean \pm SD of three representative experiments. ${ }^{* *} P<0.01$; ${ }^{* * *} P<0.001 ;{ }^{* * * *} P<0.0001$.

schematic (Fig. 5a). We cultured 4T1 cells in the above-generated BMDM-CM for $24 \mathrm{~h}$ and assayed cell migration. We found that the addition of the control M2-CM dramatically increased 4T1 cell migration, which was significantly and partially prevented in the $15 \mathrm{w}$-treated M2-CM (Fig. 5b). To further study the role of SMS2 in TNBC metastasis in vivo, we established a murine lung metastasis model by injecting 4T1-luc cells via the tail vein of BALB/c mice. Two groups of female mice were orally administered vehicle or $20 \mathrm{mg} / \mathrm{kg} \mathrm{15w}$ once a day. After 2 weeks, the plasma SM level in $15 \mathrm{w}$-treated mice was significantly reduced compared with that in vehicle-treated mice (Fig. $5 \mathrm{c}$ ). We then injected 4T1-luc cells and monitored the fluorescence intensity in the lungs 14 days later. Bioluminescent imaging showed that the $15 \mathrm{w}$-treated mice exhibited a significant impairment of lung metastatic burden compared with that of the control mice (Fig. $5 d$ ). In addition, H\&E tissue staining also revealed that the number and size of $4 \mathrm{~T} 1$ tumor foci in the lungs were significantly reduced in the $15 \mathrm{w}$-treated group compared with the control group (Fig. 5e).

Flow cytometry analysis showed that compared with the vehicle control, treatment with $15 \mathrm{w}$ did not significantly alter the total number or CD206 MFI of the peritoneal macrophages (Fig. 5f). In contrast, IHC analysis of the lung metastases showed that $15 \mathrm{w}$ treatment significantly reduced the infiltration of $\mathrm{F} 4 / 80^{+} \mathrm{CD}_{20} 6^{+} \mathrm{M} 2$ macrophages (Fig. 5g), CD4 ${ }^{+}$FOXP3 $^{+}$Tregs (Fig. 5h), and CD11b ${ }^{+} \mathrm{Gr}-$ $1^{+}$MDSCs (Fig. 5i). Overall, these results demonstrate that targeting 


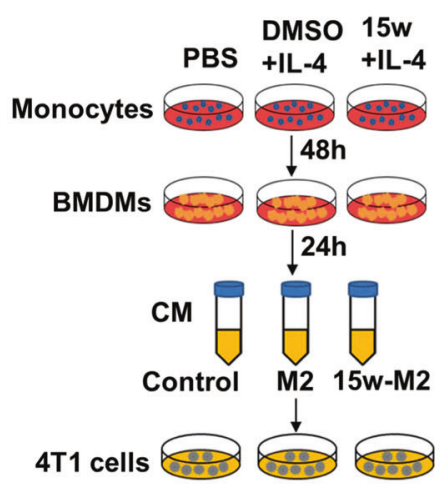

b
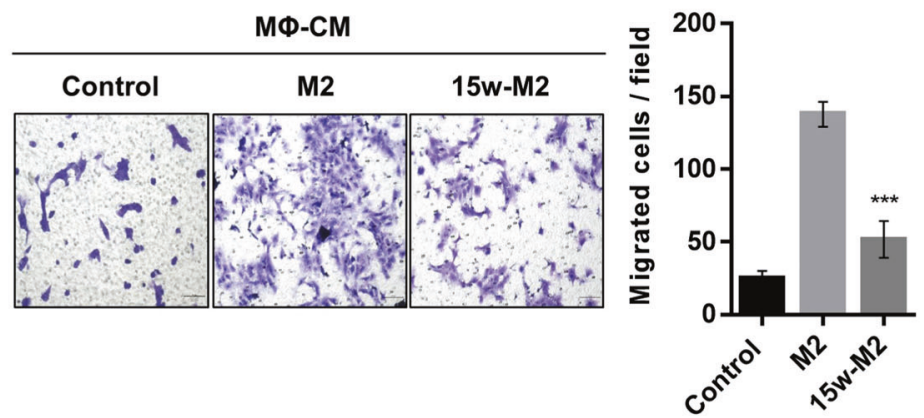

d
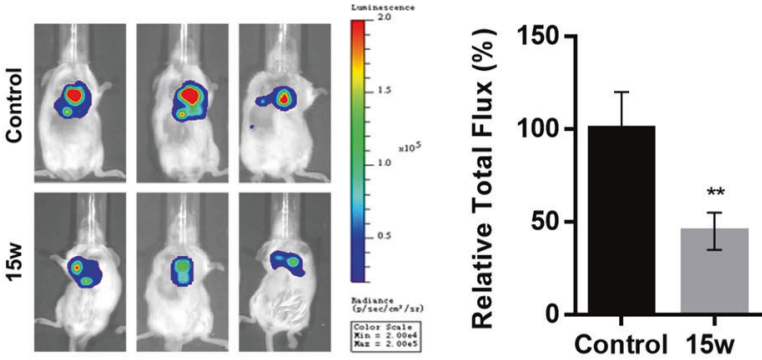

h
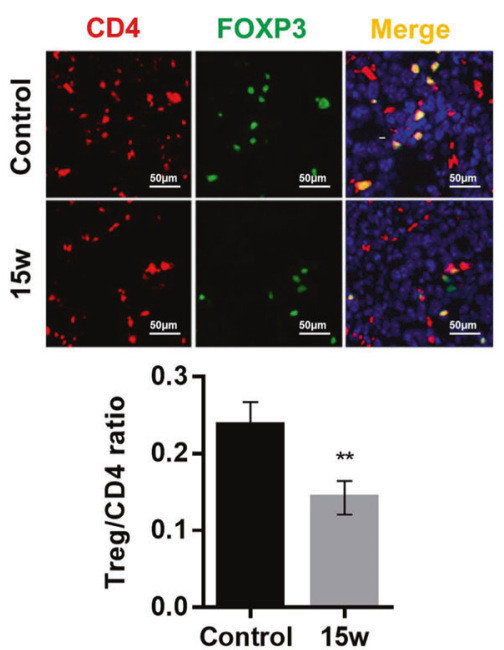
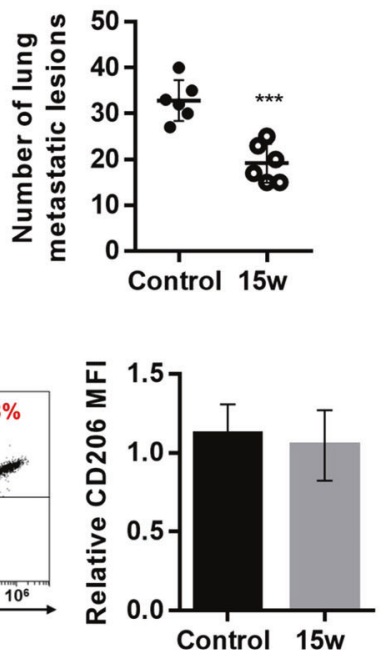

g

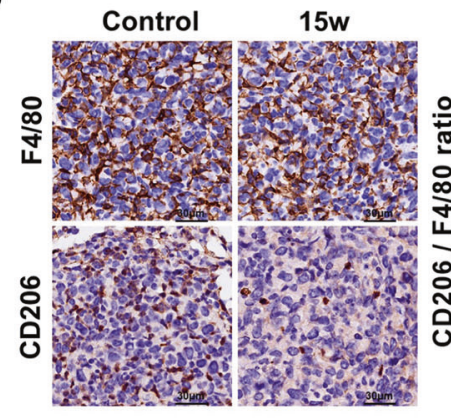

i
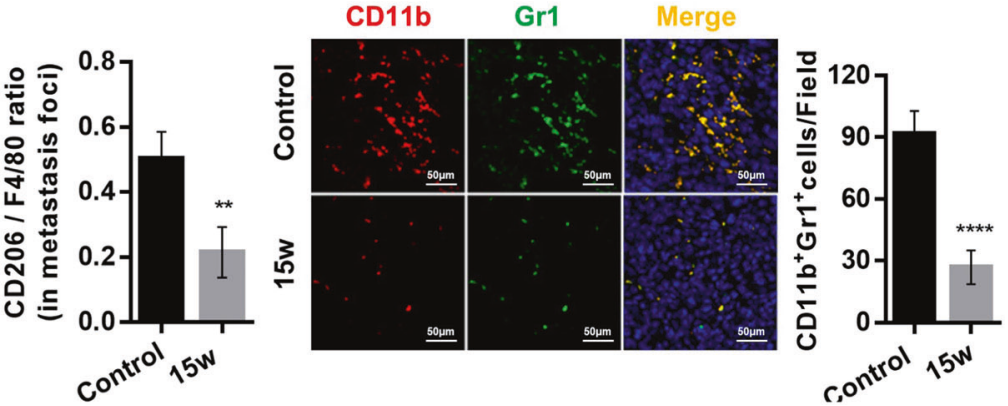

Fig. 5 SMS2 inhibitor limits the metastasis of 4T1 cells in vitro and in vivo. a Schematic diagram of the macrophage-CM collection process. b Transwell migration assays of $4 \mathrm{~T} 1$ cells. c Plasma SM levels were measured in mice after oral dosing for 14 days as described in the Methods. d Representative bioluminescent images and quantification of mice with 4T1-luc metastasis. e Representative H\&E staining and the quantification of lung metastatic burden. $\mathbf{f}$ Flow cytometry quantification of peritoneal macrophages from mice treated with or without $15 \mathrm{w}$ for 14 days. g-i Lung sections similar to those in e were analyzed by IHC staining. Representative images indicating immune cell infiltration within the metastasizing epithelial tumor compartment are shown for M2 macrophages (g), Tregs (h), and MDSCs (i). Quantification of the ratio of cell types in each lung tissue section $(n=5)$. The results are shown as the mean \pm SD. ${ }^{* *} P<0.01 ;{ }^{* * *} P<0.001 ;{ }^{* * * *} P<0.0001(n=8$ mice/group). 
a

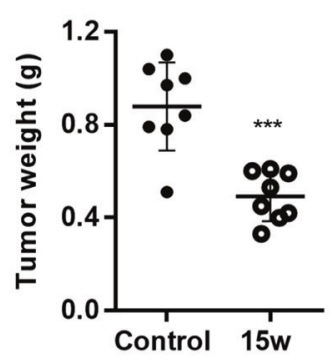

b

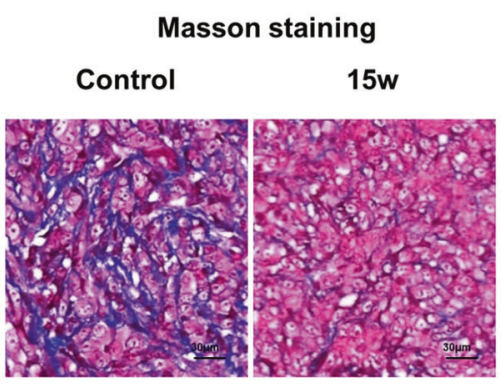

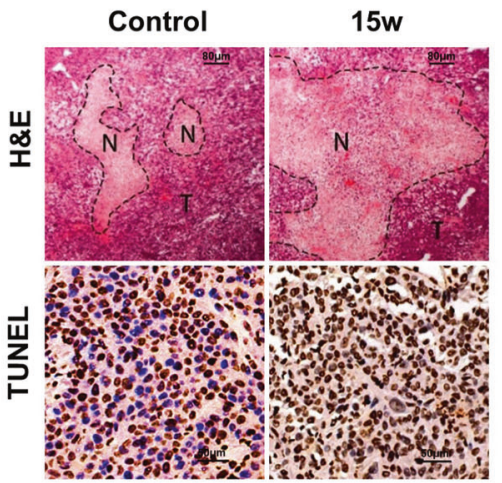
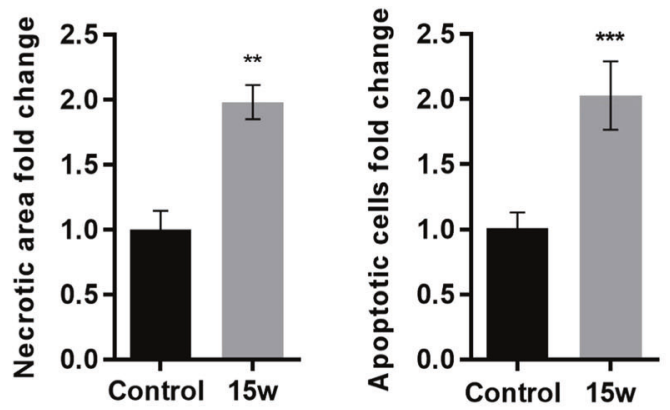

d
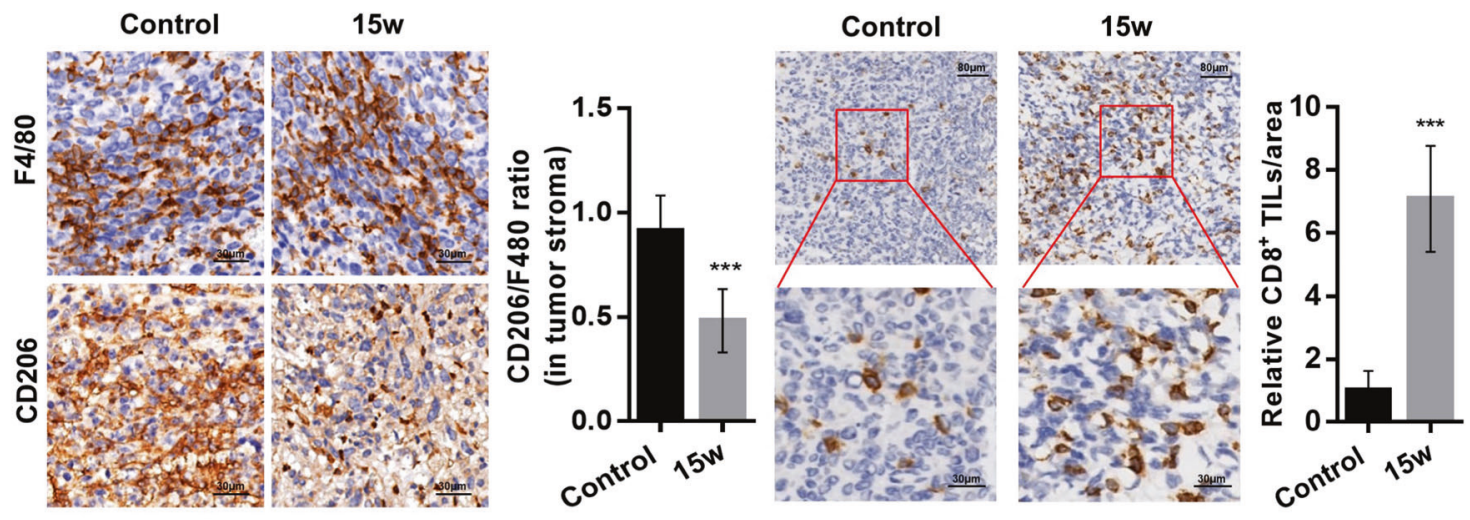

g

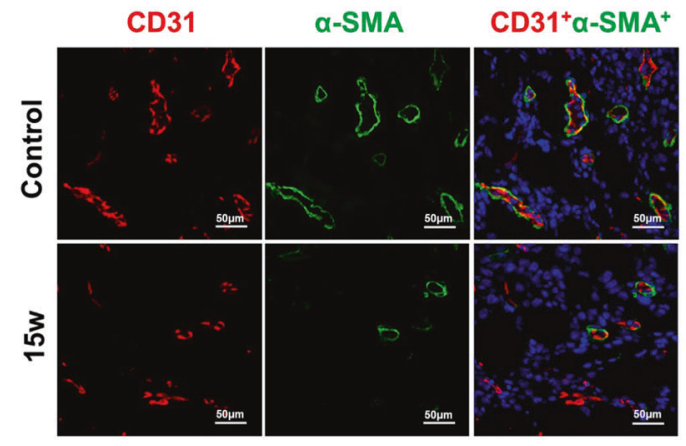

Fig. 6 SMS2 inhibitor attenuates tumor growth in a 4T1 breast cancer model. a Weights of the 4T1 primary breast tumors from treated mice. b Collagen staining in tumor tissues. c H\&E staining of tumor sections showing intensely stained necrotic areas ( $N$ necrotic tissue, $T$ tumor tissue) and TUNEL staining for apoptotic cells. d Tumor IHC staining and quantification of the CD206 to F4/80 ratio to indicate the level of M2 macrophages. e Representative IHC staining of tumor-infiltrating CD8 ${ }^{+}$lymphocytes $(\times 100$ magnification and enlarged $\times 400$ magnification). Quantifications of normalized $C D 8^{+}$lymphocytes from five fields per tumor are shown. The lumen size of the tumor vessels was analyzed by CD31 IHC (f) and CD31 and $\alpha$-SMA dual IF staining $(\mathbf{g})$. The results are shown as the mean \pm SD. ${ }^{* *} P<0.01 ;{ }^{* * *} P<0.001(n=8$ mice/group). 
SMS2 can effectively and selectively reduce M2 macrophages in the TME and promote an antitumor immunoenvironment.

Efficacy and mechanism of the SMS2 inhibitor in an orthotopic tumor growth model of breast cancer

Previous studies have suggested that M2-polarized macrophages are critical in creating an immunosuppressive environment for tumor growth $[39,40]$. We next evaluated the effect of an SMS2 inhibitor on tumor growth. 4T1 cells were orthotopically implanted in the MFPs of female BALB/c mice, treated and monitored for tumor growth for 3 weeks. As shown in Fig. $6 \mathrm{a}$, treatment with $15 \mathrm{w}$ resulted in reduced tumor growth, as indicated by a lower tumor weight compared with that of the control tumors. Various histological staining analyses revealed that the $15 \mathrm{w}$-treated tumors exhibited weak staining and diffuse distribution of collagen (Fig. 6b); the treated tumors also had markedly increased tumor necrosis and apoptosis rates compared with those in control tumors, as shown by $\mathrm{H} \& \mathrm{E}$ and TUNEL staining (Fig. $6 \mathrm{c}$ ).

We found that the infiltrated macrophages were mainly localized in the core area of tumors. Though there was no difference in the F4/80 expression of tumor-infiltrated macrophages, the expression ratio of CD206 to F4/80 in tumors of $15 \mathrm{w}$ treated mice was significantly lower than that in control tumors (Fig. 6d). Given the central role of $\mathrm{CD}^{+} \mathrm{T}$ lymphocytes in tumor immunity, we next assessed CD8 expression in tumors. As shown in Fig. 6e, IHC staining highlighted an abundant amount of $\mathrm{CD} 8^{+}$ $T$ cells infiltrating tumors of the $15 \mathrm{w}$-treated group.

We also evaluated tumor vasculature in both groups of experimental tumors. Treatment with $15 \mathrm{w}$ resulted in reduced tumor vessel density and vessel lumen size compared with that of vehicle control tumors (Fig. 6f). As vessel integrity requires pericyte coverage of vascular sprouts for stabilization and maturation [41], we performed colocalization analysis of pericytes (a-SMA) and endothelial cells (CD31). There was significantly reduced pericyte coverage on the tumor vessels of $15 \mathrm{w}$-treated mice (Fig. 6g). Collectively, these results demonstrate that the SMS2 inhibitor can effectively prevent tumor vessel maturation, which leads to a poorly perfused vasculature in tumors.

\section{DISCUSSION}

SMS2 is the key enzyme regulating SM content in plasma membrane lipid rafts, which is closely related to membrane receptor-mediated signal transduction [42]. Previous studies have shown that SMS2 deficiency can attenuate atherosclerosis by inhibiting macrophage-derived foam cell formation and macrophage inflammatory responses [1, 43]. Recently, TAM-targeted breast cancer intervention strategies have achieved great progress in numerous preclinical studies [44, 45]. In this study, we focused on exploring the participation of SMS2 in breast cancer-infiltrated TAMs, which are mostly M2-like macrophages. We analyzed the relapse-free survival of breast cancer patients by using Kaplan-Meier plotter and found that high SMS2 expression is associated with a worse prognosis than low SMS2 expression. Database analysis of 522 tumor samples from the TCGA revealed that the SMS2 level is significantly higher in basal-like breast cancer than in luminal-like breast cancer. Moreover, we found that elevated SMS2 expression is associated with high levels of M2polarized macrophage markers, suggesting that high expression of SMS2 is associated with a high density of M2-polarized macrophage infiltration in basal-like breast tumors. Based on this hypothesis, we focused on investigating the role of SMS2 in a preclinical model of TNBC. Our in vitro results demonstrated that SMS2 knockout or SMS2 enzyme inhibiton can effectively attenuate macrophage $M 2$ polarization. The CM from TNBC cells can effectively stimulate BMDMs to differentiate into M2-polarized macrophages, while the SMS2 inhibitor can markedly attenuate this process. The tumor-CM we collected contains multiple anti- inflammatory cytokines, which might explain why macrophages can be differentiated into the $M 2$ rather than the $M 1$ phenotype and why SMS2 is involved in this process. Similarly, we found that there was no difference in the phenotype of mouse peritoneal macrophages after oral administration of vehicle or SMS2 inhibitor for two weeks. However, we consistently observed that the infiltration of M2-polarized macrophages in the tumor stroma was substantially attenuated in tumor-bearing mice treated with the SMS2 inhibitor. These results indicate that the regulatory role of SMS2 in macrophage differentiation toward the M2 phenotype can be initiated when macrophages are exposed to stimulators in the TME. In the future, we plan to study additional human TNBC cell lines (such as MDA-MB-468, Hs578T, and BT549) to study the role of SMS2-TAMs in the growth and metastasis of TNBC.

In addition to the suppressed tumor growth after SMS2 inhibitor treatment, we also observed that the tumor vessels were narrowed in diameter, and the density of pericyte-encased mature vessels was significantly reduced. This result is in line with previous studies showing that TAMs control blood vessel morphogenesis in tumors $[46,47]$. In a mouse model of hematogenous metastasis, the number of M2 macrophages was relatively lower in the metastatic foci of SMS2 inhibitor-treated mice than in those of control mice. Interestingly, we also found that the overall recruitment of MDSCs and Tregs cells was considerably lower, while $\mathrm{CD} 8^{+} \mathrm{T}$ cell infiltration was profoundly increased in SMS2 inhibitor-treated mice. These results are consistent with those of previous studies [48-50]. Though we found the critical role of SMS2 in macrophage M2 polarization and tumor suppression, we still need to obtain deeper insight into the mechanism of SMS2-mediated macrophage polarization. In addition, the regulatory role of SMS2 on other immune cells and the whole immune microenvironment deserves further investigation.

To the best of our knowledge, our study is the first to demonstrate the participation of SMS2 in the process of macrophage M2 polarization and to apply an SMS2 inhibitor in antitumor immunotherapy. Though the mechanism by which SMS2 regulates M2 macrophages or other immune cells requires further examination, our findings may provide new insight into SMS2-targeted immunotherapy for TNBC.

\section{ACKNOWLEDGEMENTS}

This study was supported by the Shanghai Municipal Committee of Science and Technology (No. 17431902300), the National Science \& Technology Major Project "Key New Drug Creation and Manufacturing Program" of China (No. 2018ZX09711002), and General Program of National Natural Science Foundation of China (No. 31770864)

\section{AUTHOR CONTRIBUTIONS}

Conception and design: YD, JBD, and KY. Development of methodology: YD, XCJ, JBD, and KY. Acquisition of data: $Y D, J C H, S H H, B L, T B D$, and JBD. Analysis and interpretation of data: $Y D, X C J, J B D$, and KY. Writing, review, and/or revision of the manuscript: $Y D, X C J, J B D$, and KY. Administrative, technical, or material support: JTY, MGM, DYY, and LZ. Study supervision: YD, JBD, and KY.

\section{ADDITIONAL INFORMATION}

The online version of this article (https://doi.org/10.1038/s41401-020-0419-1) contains supplementary material, which is available to authorized users.

Competing interests: The authors declare no competing interests.

\section{REFERENCES}

1. Denkert C, Liedtke $C$, Tutt A, von Minckwitz G. Molecular alterations in triplenegative breast cancer-the road to new treatment strategies. Lancet. 2017; 389:2430-42.

2. Bray F, Ferlay J, Soerjomataram I, Siegel RL, Torre LA, Jemal A. Global cancer statistics 2018: GLOBOCAN estimates of incidence and mortality worldwide for 36 cancers in 185 countries. CA Cancer J Clin. 2018;68:394-424. 
3. Longo R, Torino F, Gasparini G. Targeted therapy of breast cancer. Curr Pharmacol Des. 2007:13:497-517.

4. Pal SK, Childs BH, Pegram M. Triple negative breast cancer: unmet medical needs. Breast Cancer Res Treat. 2011;125:627-36.

5. Liedtke C, Mazouni C, Hess KR, Andre F, Tordai A, Mejia JA, et al. Response to neoadjuvant therapy and long-term survival in patients with triple-negative breast cancer. J Clin Oncol. 2008;26:1275-81.

6. Echeverria GV, Ge Z, Seth S, Zhang X, Jeter-Jones S, Zhou X, et al. Resistance to neoadjuvant chemotherapy in triple-negative breast cancer mediated by a reversible drug-tolerant state. Sci Transl Med. 2019;11:eaav0936.

7. Chaudhary LN, Wilkinson $\mathrm{KH}$, Kong A. Triple-negative breast cancer: who should receive neoadjuvant chemotherapy? Surg Oncol Clin N Am. 2018;27:141-53.

8. Ruffell B, Coussens LM. Macrophages and therapeutic resistance in cancer Cancer Cell. 2015;27:462-72.

9. Casey SC, Amedei A, Aquilano K, Azmi AS, Benencia F, Bhakta D, et al. Cancer prevention and therapy through the modulation of the tumor microenvironment. Semin Cancer Biol. 2015;35:S199-223.

10. Deepak KGK, Vempati R, Nagaraju GP, Dasari VR, S N, Rao DN, et al. Tumor microenvironment: challenges and opportunities in targeting metastasis of triple negative breast cancer. Pharmacol Res. 2020;153:104683.

11. Lewis CE, Pollard JW. Distinct role of macrophages in different tumor microenvironments. Cancer Res. 2006;66:605-12.

12. Williams CB, Yeh ES, Soloff AC. Tumor-associated macrophages: unwitting accomplices in breast cancer malignancy. NPJ Breast Cancer. 2016;2:15025. pii

13. Linde N, Casanova-Acebes M, Sosa MS, Mortha A, Rahman A, Farias E, et al. Macrophages orchestrate breast cancer early dissemination and metastasis. Nat Commun. 2018;9:21.

14. Mantovani A, Sozzani S, Locati M, Allavena P, Sica A. Macrophage polarization: tumor-associated macrophages as a paradigm for polarized M2 mononuclear phagocytes. Trends Immunol. 2002;23:549-55.

15. Chen Y, Zhang S, Wang Q, Zhang X. Tumor-recruited M2 macrophages promote gastric and breast cancer metastasis via M2 macrophage-secreted CHI3L1 protein. J Hematol Oncol. 2017;10:36

16. Mills CD, Kincaid K, Alt JM, Heilman MJ, Hill AM. M-1/M-2 macrophages and the Th1/Th2 paradigm. J Immunol. 2000;164:6166-73.

17. Quail DF, Joyce JA. Microenvironmental regulation of tumor progression and metastasis. Nat Med. 2013;19:1423-37.

18. Xu M, Liu M, Du X, Li S, Li H, Li X, et al. Intratumoral delivery of IL-21 overcomes anti-Her2/Neu resistance through shifting tumor-associated macrophages from M2 to M1 phenotype. J Immunol. 2015;194:4997-5006.

19. Anfray C, Ummarino A, Andon FT, Allavena P. Current strategies to target tumor-associated-macrophages to improve anti-tumor immune responses. Cells. 2019;9:E46.

20. Shiao SL, Ruffell B, DeNardo DG, Faddegon BA, Park CC, Coussens LM. TH2polarized $\mathrm{CD}^{+} \mathrm{T}$ cells and macrophages limit efficacy of radiotherapy. Cancer Immunol Res. 2015;3:518-25.

21. Zhang QW, Liu L, Gong CY, Shi HS, Zeng YH, Wang XZ, et al. Prognostic significance of tumor-associated macrophages in solid tumor: a meta-analysis of the literature. PLoS One. 2012;7:e50946.

22. Medrek C, Ponten F, Jirstrom K, Leandersson K. The presence of tumor associated macrophages in tumor stroma as a prognostic marker for breast cancer patients. BMC Cancer. 2012;12:306.

23. Hollmen M, Karaman S, Schwager S, Lisibach A, Christiansen AJ, Maksimow M et al. G-CSF regulates macrophage phenotype and associates with poor overall survival in human triple-negative breast cancer. Oncoimmunology. 2016;5: e1115177.

24. Jeong H, Hwang I, Kang SH, Shin HC, Kwon SY. Tumor-associated macrophages as potential prognostic biomarkers of invasive breast cancer. J Breast Cancer. 2019;22:38-51.

25. Yuan ZY, Luo RZ, Peng RJ, Wang SS, Xue C. High infiltration of tumor-associated macrophages in triple-negative breast cancer is associated with a higher risk of distant metastasis. Onco Targets Ther. 2014;7:1475-80.

26. Tiainen S, Masarwah A, Oikari S, Rilla K, Hamalainen K, Sudah M, et al. Tumor microenvironment and breast cancer survival: combined effects of breast fat, $\mathrm{M} 2$ macrophages and hyaluronan create a dismal prognosis. Breast Cancer Res Treat. 2020;179:565-75.

27. Kolesnick RN. Sphingomyelin and derivatives as cellular signals. Prog Lipid Res. 1991;30:1-38.
28. Simons K, Toomre D. Lipid rafts and signal transduction. Nat Rev Mol Cell Biol. 2000;1:31-9.

29. Zama K, Mitsutake S, Okazaki T, Igarashi Y. Sphingomyelin in microdomains of the plasma membrane regulates amino acid-stimulated mTOR signal activation. Cell Biol Int. 2018;42:823-31.

30. Liu J, Zhang H, Li Z, Hailemariam TK, Chakraborty M, Jiang K, et al. Sphingomyelin synthase 2 is one of the determinants for plasma and liver sphingomyelin levels in mice. Arterioscler Thromb Vasc Biol. 2009;29:850-6.

31. Yeang C, Ding T, Chirico WJ, Jiang XC. Subcellular targeting domains of sphingomyelin synthase 1 and 2. Nutr Metab. 2011;8:89.

32. Liu J, Huan C, Chakraborty M, Zhang H, Lu D, Kuo MS, et al. Macrophage sphingomyelin synthase 2 deficiency decreases atherosclerosis in mice. Circ Res. 2009;105:295-303.

33. Hailemariam TK, Huan C, Liu J, Li Z, Roman C, Kalbfeisch M, et al. Sphingomyelin synthase 2 deficiency attenuates NFkappaB activation. Arterioscler Thromb Vasc Biol. 2008;28:1519-26.

34. Mo M, Yang J, Jiang XC, Cao Y, Fei J, Chen Y, et al. Discovery of 4-benzyloxybenzo [d]isoxazole-3-amine derivatives as highly selective and orally efficacious human sphingomyelin synthase 2 inhibitors that reduce chronic inflammation in $\mathrm{db} / \mathrm{db}$ mice. J Med Chem. 2018;61:8241-54.

35. Cancer Genome Atlas N. Comprehensive molecular portraits of human breast tumours. Nature. 2012;490:61-70.

36. Wan $X$, Zheng $X$, Pang X, Pang Z, Zhao J, Zhang Z, et al. Lapatinib-loaded human serum albumin nanoparticles for the prevention and treatment of triple-negative breast cancer metastasis to the brain. Oncotarget. 2016;7:34038-51.

37. Huang T, Li X, Hu S, Zhao B, Chen P, Liu X, et al. Analysis of fluorescent ceramide and sphingomyelin analogs: a novel approach for in vivo monitoring of sphingomyelin synthase activity. Lipids. 2014;49:1071-9.

38. Zhang X, Li Q, Zhao H, Ma L, Meng T, Qian J, et al. Pathological expression of tissue factor confers promising antitumor response to a novel therapeutic antibody SC1 in triple negative breast cancer and pancreatic adenocarcinoma. Oncotarget. 2017;8:59086-102.

39. Yeung OW, Lo CM, Ling CC, Qi X, Geng W, Li CX, et al. Alternatively activated (M2) macrophages promote tumour growth and invasiveness in hepatocellular carcinoma. J Hepatol. 2015;62:607-16.

40. Sossey-Alaoui K, Pluskota E, Bialkowska K, Szpak D, Parker Y, Morrison CD, et al. Kindlin-2 regulates the growth of breast cancer tumors by activating CSF-1mediated macrophage infiltration. Cancer Res. 2017;77:5129-41.

41. Raza A, Franklin MJ, Dudek AZ. Pericytes and vessel maturation during tumor angiogenesis and metastasis. Am J Hematol. 2010;85:593-8.

42. Breslow DK, Weissman JS. Membranes in balance: mechanisms of sphingolipid homeostasis. Mol Cell. 2010;40:267-79.

43. Lou B, Dong J, Li Y, Ding T, Bi T, Li Y, et al. Pharmacologic inhibition of sphingomyelin synthase (SMS) activity reduces apolipoprotein-B secretion from hepatocytes and attenuates endotoxin-mediated macrophage inflammation. PLoS One. 2014;9:e102641.

44. Pyonteck SM, Akkari L, Schuhmacher AJ, Bowman RL, Sevenich L, Quail DF, et al CSF-1R inhibition alters macrophage polarization and blocks glioma progression. Nat Med. 2013;19:1264-72.

45. Qian BZ, Li J, Zhang H, Kitamura T, Zhang J, Campion LR, et al. CCL2 recruits inflammatory monocytes to facilitate breast-tumour metastasis. Nature. 2011; 475:222-5.

46. Lin EY, Li JF, Gnatovskiy L, Deng Y, Zhu L, Grzesik DA, et al. Macrophages regulate the angiogenic switch in a mouse model of breast cancer. Cancer Res. 2006;66:11238-46.

47. Leek RD, Lewis CE, Whitehouse R, Greenall M, Clarke J, Harris AL. Association of macrophage infiltration with angiogenesis and prognosis in invasive breast carcinoma. Cancer Res. 1996;56:4625-9.

48. Savage ND, de Boer T, Walburg KV, Joosten SA, van Meijgaarden K, Geluk A, et al. Human anti-inflammatory macrophages induce Foxp $3^{+} \mathrm{GITR}^{+} \mathrm{CD} 25^{+}$ regulatory $T$ cells, which suppress via membrane-bound TGFbeta-1. J Immunol. 2008;181:2220-6.

49. Curiel TJ, Coukos G, Zou L, Alvarez X, Cheng P, Mottram P, et al. Specific recruitment of regulatory $T$ cells in ovarian carcinoma fosters immune privilege and predicts reduced survival. Nat Med. 2004;10:942-9.

50. Peranzoni E, Lemoine J, Vimeux L, Feuillet V, Barrin S, Kantari-Mimoun $C$, et al. Macrophages impede CD8 T cells from reaching tumor cells and limit the efficacy of anti-PD-1 treatment. Proc Natl Acad Sci U S A. 2018;115·E4041-50. 